\title{
Hardware Development Process for Human Research Facility Applications
}

\author{
Liz Bauer \\ National Aeronautics and Space Administration, Johnson Space Center, 2101 NASA Road 1, \\ Mail stop: EA5, Houston, TX, 77058 \\ (281)483-7304,ebauer@ems.jsc.nasa.gov
}

\section{EXTENDED ABSTRACT}

The simple goal of the Human Research Facility (HRF) is to conduct human research experiments on the International Space Station (ISS) astronauts during long-duration missions. This is accomplished by providing integration and operation of the necessary hardware and software capabilities. A typical hardware development flow consists of five stages: functional inputs and requirements definition, market research, design life cycle through hardware delivery, crew training, and mission support. The purpose of this presentation is to guide the audience through the early hardware development process: requirement definition through selecting a development path. Specific HRF equipment is used to illustrate the hardware development paths.

The source of hardware requirements is the science community and HRF program. The HRF Science Working Group, consisting of scientists from various medical disciplines, defined a basic set of equipment with functional requirements. This established the performance requirements of the hardware. HRF program requirements focus on making the hardware safe and operational in a space environment. This includes structural, thermal, human factors, and material requirements. Science and HRF program requirements are defined in a hardware requirements document which includes verification methods. Once the hardware is fabricated, requirements are verified by inspection, test, analysis, or demonstration. All data is compiled and reviewed to certify the hardware for flight. Obviously, the basis for all hardware development activities is requirement definition.

Full and complete requirement definition is ideal prior to initiating the hardware development. However, this is generally not the case, but the hardware team typically has functional inputs as a guide. The first step is for engineers to conduct market research based on the functional inputs provided by scientists. Commercially available products are evaluated against the science requirements as well as modifications needed to meet program requirements. Options are consolidated and the hardware development team reaches a hardware development decision point. Within budget and schedule constraints, the team must decide whether or not to complete the hardware as an in-house, subcontract with vendor, or commercial-off-the-shelf (COTS) development. An in-house development indicates NASA personnel or a contractor builds the hardware at a NASA site. A subcontract development is completed off-site by a commercial company. A COTS item is a vendor product available by ordering a specific part number. The team evaluates the pros and cons of each development path. For example, in-house developments utilize existing corporate knowledge regarding how to build equipment for use in space. However, technical expertise would be required to fully understand the medical equipment capabilities, such as for an ultrasound system. It may require additional time and funding to gain the expertise that commercially exists. The major benefit of subcontracting a hardware development is the product is delivered as an end-item and commercial expertise is utilized. On the other hand, NASA has limited control over schedule delays. The final option of COTS or modified COTS equipment is a compromise between in-house and subcontracts. A vendor product may exist that meets all functional requirements but requires in-house modifications for successful 
operation in a space environment. The HRF utilizes equipment developed using all of the paths described: inhouse, subcontract, and modified COTS.

The Workstation drawer is an example of an in-house development. It is a key computational element in the data system architecture. The overall system consists of the rack drawer, display, keyboard, archive media, and cables. The Workstation provides data collection, archive, downlink, display, video processing, graphics accelerator, user interface, and rack interface. It provides a platform for installing and executing HRF software to perform experiment procedures. The drawer features a $200 \mathrm{MHz}$ Pentium CPU, 256 Kbytes RAM, and Windows NT operating system. It provides the following electrical and mechanical data interfaces: Ethernet (10Base-T), RS232C, RS-422, floppy drive, RGB video, audio in/out, $32 \mathrm{~A} / \mathrm{D}$ and $2 \mathrm{D} / \mathrm{A}$ channels, 16 digital I/O channels, auxiliary $28 \mathrm{Vdc}$, and PCMCIA and SCSI hard drive capabilities. Lockheed Martin (LM) integrated the COTS and in-house designed components, boards, and cabling into a rack drawer. LM was responsible for the design, layout, and functionality. The drawer was assembled and tested using NASA Johnson Space Center (JSC) facilities. For Workstation accessories, modified COTS and subcontract equipment are used. The keyboard and archive media are catalog available items needing in-house additions of labels and Velcro. The team issued a subcontract for the display. A vendor item did not exist which would meet the shared Workstation and Ultrasound requirements. Although the Workstation system utilizes COTS and subcontract hardware, the technical expertise is located and maintained at JSC because of the in-house drawer and overall system design. This aspect will be highly beneficial given its integral role in the HRF.

An example of a subcontract is the HRF Gas Analyzer System for Metabolic Analysis of Physiology (GASMAP). The system is used for periodic assessment of crew aerobic capacity. The GASMAP monitors volume and flow of inspired and expired gas, calculated by integration of instantaneous flow multiplied by fractional gas concentration. Measurement and analysis of the following quantities analyze metabolic activity: temperature of gas inspired, volume of inspired and expired gas, frequency of respiration, and ambient barometric pressure. The vendor is General Electric/Marquette Medical Systems (GE/MMS). The rack drawer was provided to GE/MMS who then integrated the analyzer equipment, coded software, and delivered an end-item to NASA.

The HRF utilizes a significant amount of COTS equipment needing minor to major in-house modifications. As previously mentioned, the Workstation keyboard is an example of minor modifications. Another example is the Portable Computer System (PCS). It provides support for stand-alone experiment control and data collection. The PCS displays high-resolution color images and text. It is a software/data interface and storage device for the rack, drawers, and experiment equipment. The COTS unit is an IBM $760 \mathrm{XD}$. In-house modifications include replacing the lithium battery with a NiMH one and using MIL SPEC connectors for durability. These are minor modifications in comparison to the HRF Ultrasound that required major in-house modifications. The Ultrasound is capable of high resolution imaging in a wide range of applications, both research and diagnostic. It is used to perform non-invasive, portable radiology with cardiac, abdominal, vascular, and musculoskeletal applications. It allows for stereo, audio output, and voice annotation by the ISS crew. The COTS unit and keyboard are Advanced Technology Laboratory (ATL) products. Lockheed Martin completed the in-house modifications at JSC. The ATL unit layout was turned on its side and repackaged into a 16 panel unit rack drawer. A power module, 2 fans, and analog recorder were added to the drawer. The ATL keyboard was repackaged into a metal case to limit offgas products. Since a significant amount of modifications were made in-house, ATL and Lockheed Martin share the Ultrasound technical expertise. The Ultrasound system is an excellent example of how COTS equipment is modified for space flight.

The HRF utilizes various medical instruments and computer equipment. The starting point for hardware development is requirement definition and commercial research. The hardware development path varies among in-house (Workstation), subcontract with vendor (GASMAP), and modified COTS (PCS and Ultrasound) developments. NASA and commercial industries have a strong relationship because NASA supports commercial upgrades and industry supports advanced applications. This relationship will continue to expand over the next decade as HRF supports life sciences research aboard the ISS. 